Ann. Abeille, I963, 6 (4), 277-30I.

\title{
ÉTUDE EXPÉRIMENTALE DE LA TRAPPE A POLLEN EN POSITION SUPÉRIEURE
}

\author{
P. LAVIE et J. FRESNAYE \\ Station expérimentale d'Apiculture, \\ Centre de Recherches agronomiques du Sud-Est, Montfavet (Vaucluse)
}

\section{SOMMAIRE}

Le présent mémoire est une étude de la récolte du pollen au moyen de trappes placées à la partie supérieure des ruches. Les expériences ont été réalisées sur 40 colonies d'abeilles dans des garrigues à Cistes du sud-est de la France. Les principaux résultats obtenus sont les suivants ;

$\left.I^{\circ}\right)$ l'influence de la surface libre du passage à l'entrée de la ruche sur la récolte de pollen est prépondérante. Plus le passage est restreint, plus la récolte est importante. La récolte optimum (et non maximum) est obtenue avec 3 rangées d'ouvertures dans le piège;

$\left.2^{\circ}\right)$ une ouverture supplémentaire libre, sur la face postérieure de la trappe, augmente la récolte du pollen ;

$3^{\circ}$ ) la récolte du pollen par les différentes colonies est pratiquement proportionnelle à l'étendue de leur couvain. Les ruches orphelines récoltent du pollen, mais en très faibles quantités ;

$4^{\circ}$ ) parmi les différents facteurs météorologiques, le vent nous a paru présenter une influence prédominante ;

$5^{\circ}$ ) I'influence des prélèvements de pollen pendant 4 o jours sur le développement du couvain et sur le poids des ruches s'est révélée pratiquement nulle. Le couvain continue à s'étendre et le poids n'est pas affecté, sauf toutefois dans certains cas limités ;

$\left.6^{\circ}\right)$ l'abeille utilise les plantes communes, mais choisit cependant certains pollens relativement rares mais très attractifs et très nutritifs ;

$7^{\circ}$ ) les récoltes peuvent être quantitativement beaucoup plus importantes que ne l'ont signalé nos prédécesseurs.

En conclusion, nous donnons quelques conseils pratiques pour améliorer la rentabilité de la récolte de pollen.

\section{INTRODUC'TION}

La récolte du pollen par l'homme, grâce à l'abeille et au moyen des trappes à pollen, est une activité d'acquisition récente. En effet, il n'y a pas trente ans que les premières trappes à pollen ont été construites en Amérique. Encore faut-il préciser que ces premiers appareils furent utilisés uniquement par les chercheurs et non par les apiculteurs. Ces derniers tenant compte des recherches effectuées sur la con- 
sommation du pollen par les abeilles se sont mis parfois à récolter du pollen dans le but de compléter la nourriture des ruches déficientes. Il n'y a qu'une dizaine d'années que la pratique de la récolte du pollen s'est généralisée dans les milieux apicoles et principalement en Europe occidentale, mais d'emblée un grand nombre d'apiculteurs se sont intéressés à la question, le pollen ayant trouvé, par ailleurs, en diététique humaine des applications intéressantes. On a vu apparaître alors une multitude de modèles de pièges à pollen plus ou moins bien conçus mais tous basés sur le même principe : l'abeille chargée de pollen est obligée, pour pénétrer dans sa ruche, de traverser une grille métallique ou une plaque perforée qui arrête en partie les pelotes fixées sur ses pattes postérieures. Ces pelotes, détachées lors du passage, sont recueillies dans un tiroir à l'abri des intempéries et auquel les abeilles ne peuvent accéder. Nous n'exposerons pas plus longtemps les différentes méthodes de récolte du pollen et nous ne décrirons pas non plus les modèles de trappes utilisés en France depuis ro ans et leurs améliorations. Là n'est pas notre propos. Les lecteurs s'intéressant à cette question se reporteront notamment aux publications de CHAUvin (I955), de Chauvin et Louveaux (1956), de Louveaux (I958) et aux nombreux articles de vulgarisation parus dans la presse apicole spécialisée. Cependant, nous devons signaler que deux tendances principales existent parmi les différents modèles utilisés: la trappe à pollen inférieure et la trappe à pollen supérieure. La trappe inférieure est placé sous le corps de ruche (ou en avant du trou de vol), le tiroir de récolte étant placé plus bas que 1'ensemble de la colonie d'abeilles. La trappe supérieure, de mise au point plus récente, se place au-dessus du nid à couvain sous le toit de la ruche. Nous avons adopté la deuxième solution pour des raisons de commodité en rapport avec l'éloignement de nos ruchers et l'importance des récoltes obtenues pendant la floraison des Cistes. Ia trappe supérieure permet de ne récolter le pollen qu'une ou deux fois par semaine sans risque de fermentation. Ėn effet, le pollen se trouve rapidement déshydraté, au moins partiellement, grâce à la chaleur de la colonie d'abeilles située au-dessous de lui. Le pollen n'est jamais mouillé par l'eau de pluie, ni par l'eau de condensation de la ruche. Enfin les détritus sont rejetés par le bas de la ruche et ne tombent pas dans le tiroir de récolte. Par contre, on reproche à la trappe supérieure d'être gênante lors de la manipulation des hausses et des nourrisseurs. Cependant, depuis 4 ans, nous nous servons presque uniquement de cette trappe supérieure et nous n'avons jamais été réellement gênés dans notre travail. Én effet, l'époque de récolte de pollen dans notre région, ne coïncide ni avec la grande miellée, ni avec une période de disette. Il nous est arrivé malgré tout, durant deux années de suite, d'être obligés de poser des hausses sur les ruches possédant des trappes et l'opération ne nous a pas semblé aussi compliquée que l'ont affirmé certains apiculteurs.

Bien que la production du pollen se soit maintenant stabilisée à un niveau assez bas, nous avons considéré qu'il n'était pas sans intérêt de poursuivre les recherches sur les méthodes les plus aptes à fournir une production importante sans nuire aux colonies. Nos besoins propres en vue de l'expérimentation sont élevés et, d'autre part, nous restons persuadés que les nourrissements de printemps à base de pollen prendront de plus en plus d'importance. C'est pourquoi, dès Ig60, nous avons jugé bon d'étudier scientifiquement les problèmes posés par l'utilisation des trappes à pollen, problèmes qui sont encore loin d'avoir été résolus en totalité. Des essais préliminaires ont eu lieu sur une trentaine de ruches pour débrouiller certains problèmes 
et les résultats obtenus nous ont amenés à reprendre des eșais plus précis en I96I, au même emplacement et sur un lot de 40 ruches. Nous ne tiendrons compte dans ce travail que des essais de I96I où les relevés chiffrés sont plus complets et ceci pour la clarté de l'exposé. Mais nous devons signaler que les essais de Ig60 ont donné des résultats semblables, nous permettant ainsi de tirer des conclusions valables bien que les deux saisons aient comporté des différences tant au point de vue météorologique que du point de vue du développement de la végétation.

Dès I956, Chauvin et Louveaux avaient remarqué l'importance à l'entrée des pièges à pollen de ce qu'ils avaient appelé le " coefficient de bousculade ". Ces observations préliminaires montraient que si les abeilles se pressaient en foule lors de la traversée de la tôle perforée, elles n'avaient pas le temps de passer avec précaution en évitant de laisser tomber leurs pelotes de pollen dans les tiroirs. Quelques essais avec des trappes dont l'entrée était plus ou moins réduite en surface avaient montré que le poids de la récolte était augmenté lorsque la surface de passage était très petite. C'est sur cette idée que nous sommes partis pour notre étude de la récolte du pollen au moyen de la trappe supérieure. Nos buts principaux étaient:

$\left.\mathrm{I}^{\circ}\right)$ essayer de déterminer avec précision l'influence de la surface libre de passage à l'entrée de la ruche sur la récolte de pollen et sur le comportement de la colonie;

$2^{\circ}$ ) établir quel était le passage optimum à employer pour recueillir le maximum de pollen sans toutefois nuire au développoment des abeilles.

Les nombreuses observations et les expériences que nous avions réalisées depuis plusieurs années nous ont permis d'envisager conjointement l'étude de certains autres aspects de la récolte du pollen. C'est ainsi que nous avons recherché :

- L'infuence de la surface de couvain présente avant la pose des trappes,

- L'infuence de l'orphelinage des colonies,

- L'infuence des facteurs météorologiques,

- L'infuence de la pose des trappes sur le développement ultérieur de la colonie,

- L'origine de la possibilité que possèdent certaines ruches, de récolter de grandes quantité de pollen.

Nous avons également étudié l'influence de la pose de trappes sur la récolte ultérieure de miel, mais nous ne publierons nos résultats qu'après la répétition des expériences au cours d'une nouvelle campagne.

\section{I. - MATÉRIEI, ET MÉTHODES EXPÉRIMENTALES}

\section{A) Lieu et dates des essais}

Toutes les expériences ont été effectuées en un mâme emplacement situé dans le Gard, dans une zone de garrigues où les Cistes sont très abondants et notamment, Cistus albidus sur lequel l'abeille se polarise fortement. Les ruches sont placées dans un bois de chênes verts et de robiniers très clairsemés. Les cultures situées dans la zone de batinage des abeilles sont peu mellifères et de surface restreinte. Le pollen de Cistus albidus représente environ $60 \mathrm{p}$. 100 du pollen récolté.

Bien entendi la plupart des pollens autres que celui des cistes ont été récoltés en proportions très variables au cours des essais. Le fait de récolter un pollen dominant abondant et très attractif pour l'abeille facilite les essais et permet d'éliminer quelques causes d'erreurs. En effet, l'abondance et la régularité de la récolte supprime certains facteurs limitants pour les butineuses des différentes ruches. D'autre part, la grosseur et la forme des pelotes sont relativement régulières puisqu'il s'agit d'une mền espèce végétale; les causes d'erreur sont donc réduites et le poids du pollen tombant dans les trappes est comparable d'un lot de ruches à l'autre. 
La mise en place des ruches a eu lieu quelques jours avant la pose des trappes à pollen. Ces ruches provenaient d'un même rucher et restèrent en place pendant toute la durée de la floraison du ciste. En I 96I, les abeilles sont arrivées le I I avril ; les trappes ont été posées le I 3 avril et l'expérience a duré jusqu'au 27 mai. Les ruches ont été transhumées ensuite assez rapidement dès que toutes les mesures et tous les contrôles ont été effectués. La récolte du pollen a eu lieu du i6 avril au 26 mai 196 I inclus, soit 4 I jours.

L'analyse pollinique d'un échantillon moyen prélevé pendant toute la floraison du Ciste donne les résultats ci-après.

Cistus albidus $\ldots \ldots \ldots \ldots \ldots$
Cistus monspeliensis $\ldots \ldots \ldots$
Vitis vinifera $\ldots \ldots \ldots \ldots \ldots \ldots$
Onobrychis sativa $\ldots \ldots \ldots \ldots \ldots$
Papaver rhceas $\ldots \ldots \ldots \ldots \ldots$
Thymus $\ldots \ldots \ldots \ldots \ldots \ldots \ldots$
Quercus ilex $\ldots \ldots \ldots \ldots \ldots \ldots$
Rosacées $\ldots \ldots \ldots \ldots \ldots \ldots$
Crucifères. $\ldots \ldots \ldots \ldots \ldots \ldots$

\section{B) Matériel utilisé}

Les ruches employées étaient des "Dadant " Io cadres bien peuplées. Les quarante colonies furent divisées en 4 lots après contrôle de leur poids et de leur surface de couvain. Les ruches ont été disposées sur le même emplacement mais dispersées au milieu des arbustes de manière à éviter les effets de la dérive et le regroupement des ruches des différents lots.

Nous avons déjà signalé que les récoltes de pollen sont effectuées au moyen de trappes supérieures Les planchesI et II montrent suffisamment les différents aspects du matériel pour qu'il soit superflu d'en faire une longue description. Cependant, on remarquera un certain nombre de détails qui ont leur importance. Le volume des tiroirs est volontairement très grand car ils sont règulièrement remplis en 4 à 5 jours au moment de la pleine floraison du ciste. Le fond des tiroirs est constitué par une toile de nylon ; celle-ci laisse très bien passer la chaleur provenant de la ruche, ne rouille pas comme les toiles métalliques et se révèle solide à l'usage. Le fond de chaque trappe comporte une tôle galvanisée qui, en contact avec les abeilles, transmet directement la chaleur nécessaire au séchage partiel du pollen avant son prélèvement. La pièce essentielle de la trappe mérite quelques commentaires explicatifs : il s'agit de la grille chargée de détacher les pelotes de pollen lors du passage des abeilles. Notre grille est constituée par une tôle de $34 \mathrm{~cm}$ de long sur $6 \mathrm{~cm}$ de haut, perforée de trous circulaires réguliers de $0,5 \mathrm{~cm}$ de diamètre. Sur les $6 \mathrm{~cm}$ de hauteur se répartissent neuf rangées de 49 à 50 trous chacune permettant le passage des butineuses. Pour étudier l'influence de la surface du passage libre, nous avons masqué à l'aide d'une petite lame de contreplaqué un certain nombre de rangées d'orifices. Nous avons pris 40 trappes à pollen et nous les avons divisées en 4 lots :

$\left.\mathrm{I}^{\circ}\right)$ Io trappes présentant le passage libre sur 9 rangées (ouverture totale)

$\left.2^{\circ}\right)$ Io trappes présentant le passage libre sur 6 rangées (ouverture partielle $2 / 3$ )

$\left.3^{\circ}\right)$ Io trappes présentant le passage libre sur 3 rangées (ouverture partielle $1 / 3$ )

$4^{\circ}$ ) enfin Io trappes présentant le passage libre sur I seule rangée (ouverture réduite I/9).

\section{C) Méthodes expérimentales.}

$\left.\mathrm{I}^{\circ}\right)$ Pose des trappes. La pose des trappes à pollen a lieu le même jour sur les 40 ruches. Lors de cette opération, on ferme le trou de vol des ruches et on les retourne de $180^{\circ}$ afin que leur sortie habituelle inférieure se retrouve à l'arrière. Les grilles servant à la récolte ne sont placées que deux ou trois jours après de manière à ce que les butineuses s'habituent à circuler librement dans la trappe et à sortir par la partie supérieure de leur demeure. Les 40 trappes ont été placées par groupes de ro comme il est indiqué ci-dessus. Chacun des quatre lots comprend des colonies de forces diverses de manière que les comparaisons soient valables d'un lot à l'autre. Ce triage est très facile grâce aux mesures de surface de couvain et aux pesées effectuées auparavant. Pendant toute la durée de l'expérience, il est nécessaire de procéder à des nettoyages fréquents des tiroirs qui seraient rapidement propolisés et également des plateaux de sol. De même, il faut de temps à autre laisser sortir les mâles qui encombreraient les grilles de leurs cadavres. Si une ruche ne récolte pas de pollen, une visite s'impose car c'est le signe cẹtain d'une anomalie. L'expérience a duré, en i96I, pendant 4I jours, période au cours de laquelle nous avons effectué de nombreuses observations, récoltes et 
mesures. Nous avons étudié également l'utilité d'une libre sortie pour les abeilles en plus de celle de la trappe à pollen. Pour cela nous avons, en cours d'essai, ouvert les trous de vol habituels de la moitié des ruches. Mans chaque lot, cinç colonies sur dix ont bénéficié de cette sortie supplémentaire situéz en bas et à l'arrière.

$\left.2^{\circ}\right)$ Mesures de con'rôle. Nous avons effectué avant et après l'essai, des mesures concernant la surface de couvain et le poids de charque ruche, au moyen de deux appareils mis au point par FrESNAYE (Ig6r et Ig(62). Le poids des ruches est exprimé en kilogrammes et la surface de couvain est exprimée en points (charue point représentant $40 \mathrm{~cm}^{2}$ de couvain). Le contrôle du poids des ruches soumises à l'essai a été également réalisé à l'hivernage suivant. Kinfin, nous avous noté les colonies ayant fourni des essaims postérieurement aux essais, au cours de la méme saison.

Jes relevés météorologi|jues concernant lat région ont été pris en considération. ("est l'action directe des facteurs météorologicues pendant l'expérience qui a retenu notre attention. P'ar excmple, nous savons que de nombreux facteurs météorologiques sont parfois limitants, soit pour le butinage des abeilles, soit pour l'épanouissement des fleurs.

$3^{\prime \prime)}$ Prélévements des échantillons. Pendant toute la durée de l'expérience le pollen est prélevé ruche par ruche. Ce travail est effectué régulièrement dans les tiroirs des trappes tous les cinq jours environ en période de récolte maximale. Char jue récolte individuelle numérotée est ramenée au laboratoire et pesée. (e pollen est ensuite placé sur des clayettes dans une chambre conditionnée et l'on calcule le taux d'humidité après déshydratation pendant 24 heures. Pour simplifier, nous donnerons toujours nos résultats en poids humide, sauf indication contraire dans le texte.

\section{II. - RÉSULTATS}

\section{$\mathrm{I}^{\mathrm{o})}$ Récolte du pollen en fonction des conditions météorologiques par l'ensemble des ruches}

La récolte considérée dans son ensemble a été très satisfaisante en rg6r. Elle a dépassé largement celle de I960 qui était déjà loin d'être négligeable. Nous avons en effet récolté $200,9 \mathrm{~kg}$ de pollen au cours de la floraison des Cistes qui a duré $4 \mathrm{I}$ jours Cette récolte est celle effectuée par 37 colonies, trois ruches devenues orphelines au au cours des essais n'étant pas prises en considération. Alors qu'en rg60 (avec 30 ruches) nous avions récolté $3225 \mathrm{~g}$ de pollen en moyenne par ruche, nous en avons recueilli en Ig6I : 5430 (soit 44 I3 g poids sec). La moyenne journalière de récolte (poids sec) par ruche est de I07,5 g. La teneur moyenne en eau de ce pollen dans les tiroirs a été de $I 7,5$ p. Ioo avec des valeurs extrêmes situées entre 9,5 p. Ioo et $25 \mathrm{p}$. IoO. La figure I donne la physionomie d'ensemble de cette récolte en liaison avec quelques facteurs météorologiques. Nous n'avons jamais remarqué de baisse de rendement générale de la part des ruches normales sauf dans deux cas, par mauvais temps avec vent violent pendant plusieurs jours empêchant la sortie des butineuses et également lors de la fin de la floraison.

Nous constatons un rapport très net entre les jours de fort " mistral " et les récoltes médiocres. La période la plus typique est celle du 8 au I I mai en pleine floraison du ciste, où le mistral a souffé violemment (jusqu'à II $5 \mathrm{~km} / \mathrm{h}$ ), notre récolte du 13 mai représente environ le tiers de celle du 8 mai. Par contre, la récolte reprend ensuite et malgré de très gros orages le 18 , la récolte du I9 mai est très satisfaisante. Ėnsuite, la quantité de pollen recueillie diminue à nouveau, d'une part à cause de 3 jours de " mistral » suivi de pluie et d'autre part, à cause de la fin de la floraison des Cistes. Nous avons porté sur la figure I de nombreuses indications météorologiques qui nous semblent importantes en ce qu'elles conditionnent la sortie des butineuses. Ia pluie diurne par exemple a plus d'intérêt pour nous que la 


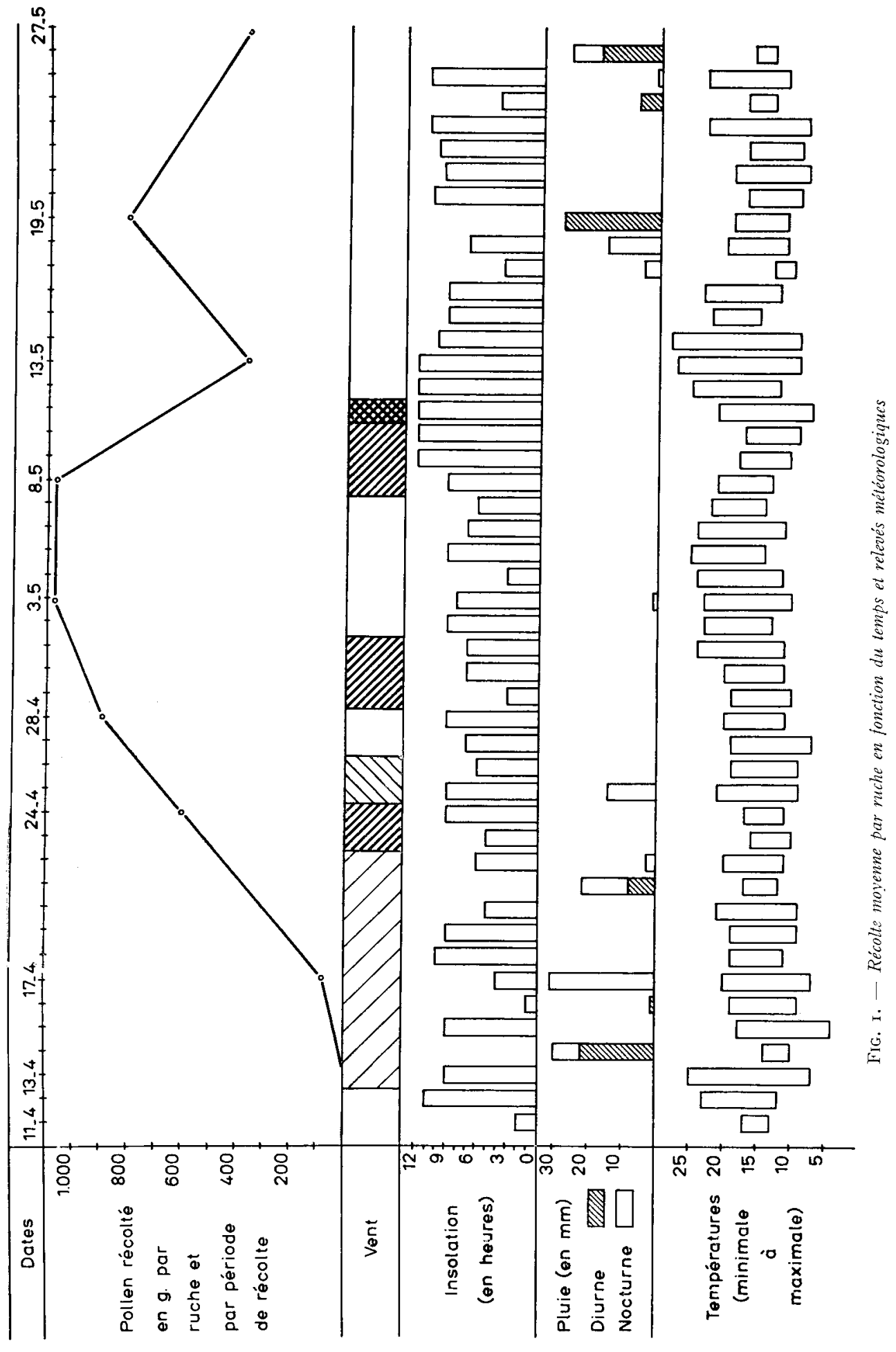


pluie totale. L'insolation en heures est aussi un facteur très important lorsqu'il est lié à la température. Nous avons noté les températures maximales et minimales qui ont toutes deux leur importance; il ne faut pas oublier à ce sujet que le Ciste est visité principalement le matin. Dès que la température et l'ensoleillement sont trop élevés, les fleurs se fanent et les pétales tombent. Cependant, il faut insister sur le fait que, dans notre région, le vent violent est le facteur limitant le plus important. Il est cependant remarquable d'avoir réalisé une récolte aussi abondante pendant les 4I jours de l'esjai et si l'on excepte la période de début et de fin de floraison, en 25 jours de pleine floraison, le 37 ruches ont récolté près de $\mathrm{I} 60 \mathrm{~kg}$ de pollen malgré une période de temps franchement mauvaise.

I'n ce qui concerne l'état des 37 ruches à la fin de l'expérience on note que :

Io) leur poids total est passé de 976 à $979 \mathrm{~kg}$ soit un gain négligeable de $3 \mathrm{~kg}$

$2^{\circ}$ ) leur surface de couvain est passée de 5906 points à 8268 points soit un gain de 382 points (soit $4, \mathrm{I} 2 \mathrm{dm}^{2}$ par ruche).

$3^{\circ)}$ nous avons retrouvé trois ruches orphelines sur quarante et ceci dans les groupes où le passage des abeilles dans la trappe était le plus libre (6 et 9 rangs de trous.)

Nous verrons d'ailleurs, ultérieurement, plus en détail les variations enregistrées dans les poids et les surface; de couvain des ruches en fonction de certains facteurs.

Il conviendrait d'ajouter également que la miellée dans notre zone de travail est faible à cette époque et que les abeilles ne font que compenser la consommation de miel nécessaire au développement de leurs colonies.

\section{$2^{\circ)}$ Récolte du pollen en fonction de la surface de passage des abeilles à l'entrée}

Connaître la récolte optimum du pollen en fonction de la surface de passage libre dans la trappe était, nous l'avons déjà dit, notre principal but. Nous avions déjà vu en Ig60 sur une trentaine de ruches que plus le passage était restreint plus la récolte était forte. Cependant, si la récolte maximum pouvait être obtenue avec par exemple, un seul rang de trous de passage libre, ne risquait-on pas d'influencer fâcheusement l'avenir de la colonie! C'est à cette question que nous avons essayé de répondre en recherchant non pas la récolte maxima de pollen, mais la récolte

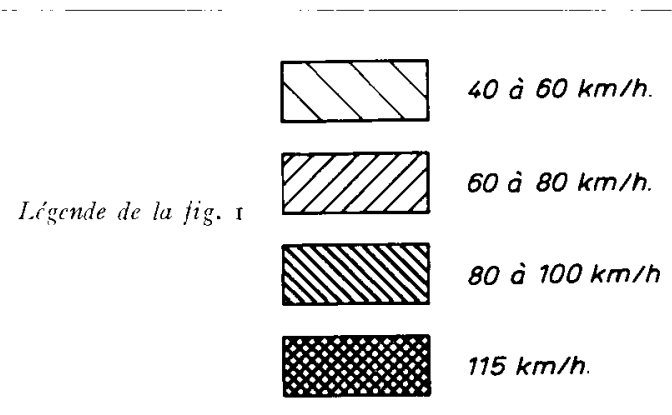


optima ne nuisant pas au développement de la ruche. Les tableaux I, 2, 3, 4 donnent en détail les résultats obtenus. On y trouve la récolte totale de chaque ruche et la récolte moyenne de chaque lot. La figure 2 donne d'autre part la récolte moyenne ce chaque groupe en fonction du temps. L'examen de tous ces résultats montre que les

\section{TABLEAU I}

Pollen ricolté au moyen des trappes comportant

1 rang de passage libre

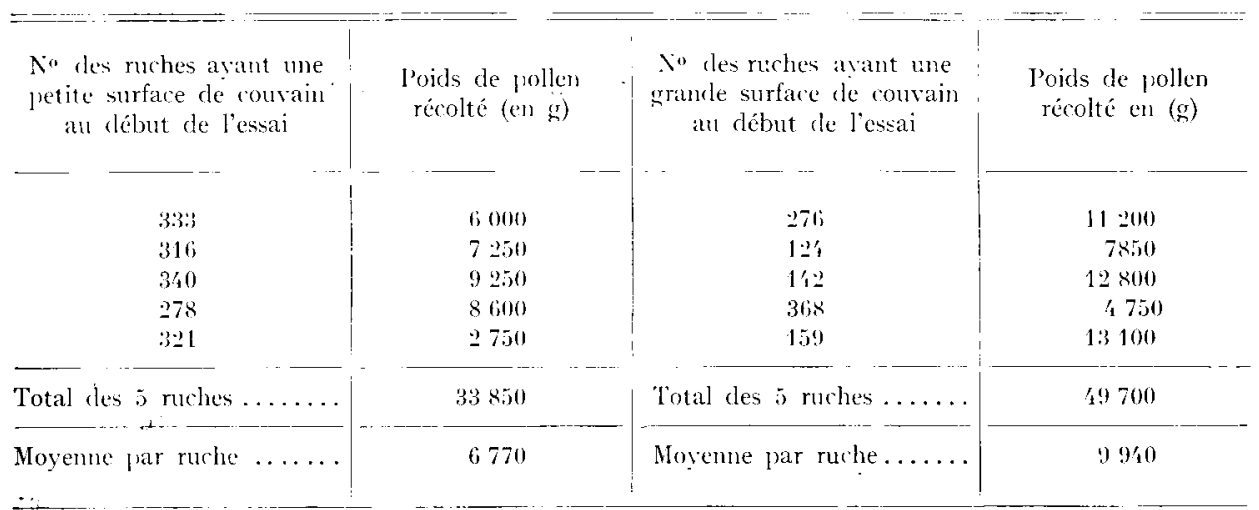

Récolte totale du lot des 10 ruches : 83550

Moyeme générale récoltée par l'ensemble du lot : 8355

$=\ldots$

TABLEAU 2

Pollen ricolte an moyen des trappes compontant 3 rangs de passage lilve

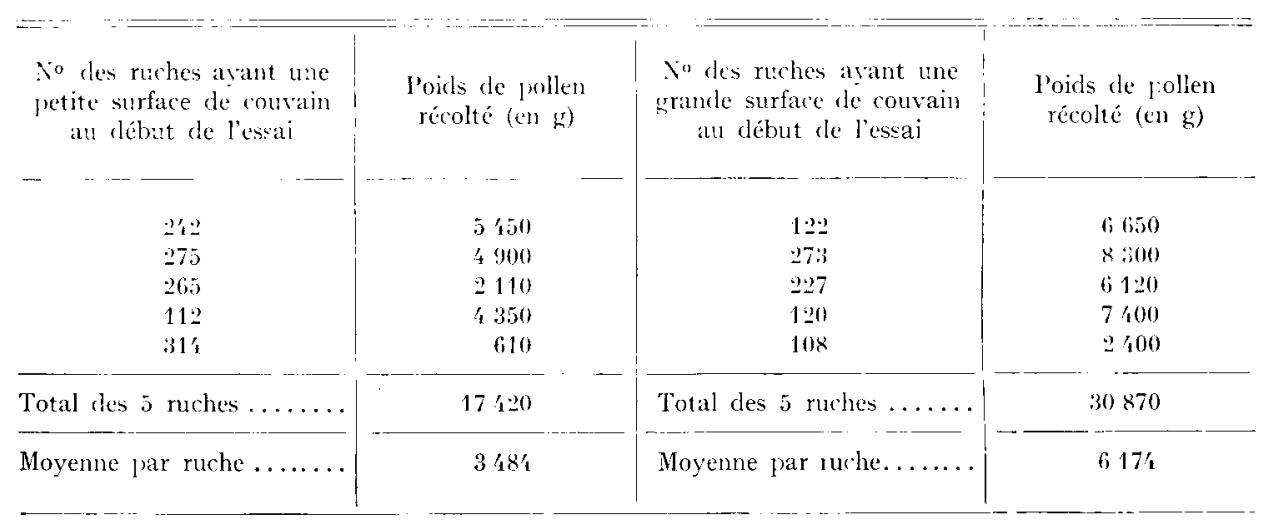

Récolte totale du lot de 10 ruches : 48 299)

Moyenne générale récoltée par l'ensemble du lot : 4829 
colonies d'abeilles disposant seulement d'un rang de trous pour le passage à l'entrée ont récolté un poids de pollen bien supérieur aux autres : $8355 \mathrm{~g}$ de moyenne par ruche. Nous avons donc comme en ig6o retrouvé les mêmes résultats concernant la récolte maximum de pollen dans les trappes. Le poids de pollen recueilli augmente lorsque le

TABLEAU 3

Pollen ricolte' au moyen des trappes comportant

6 rangs de passage libre

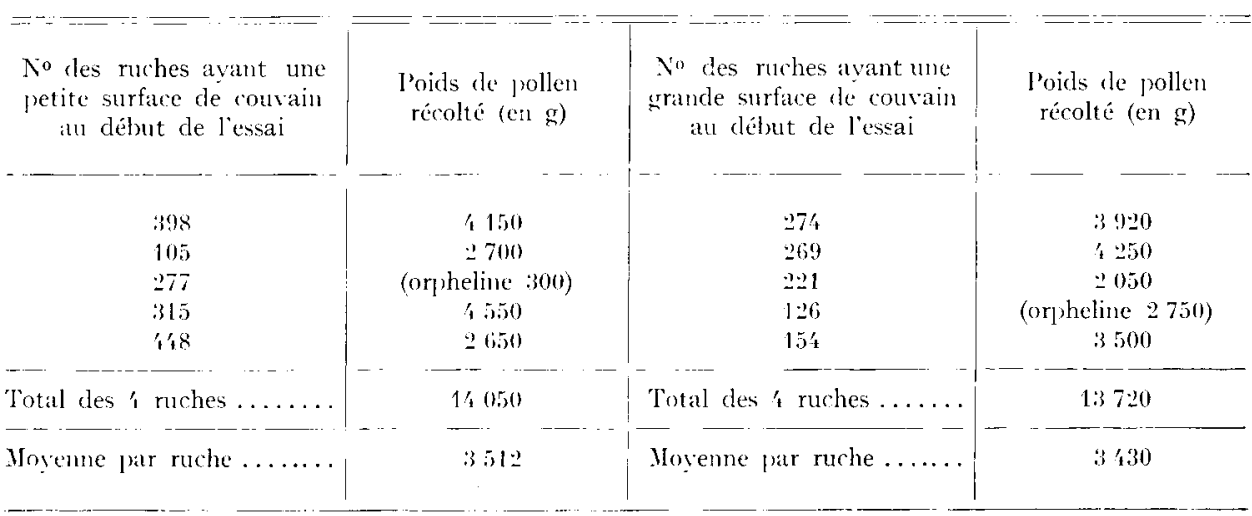

Récolte totale du lot de 8 ruches (sans les orphelines) : 27770

Moyenne générale récoltée par l'ensemble du lot : 3471

TABLEAU 4

Pollen ricolte au moyen des trappes comportant

9 rangs de passage libre

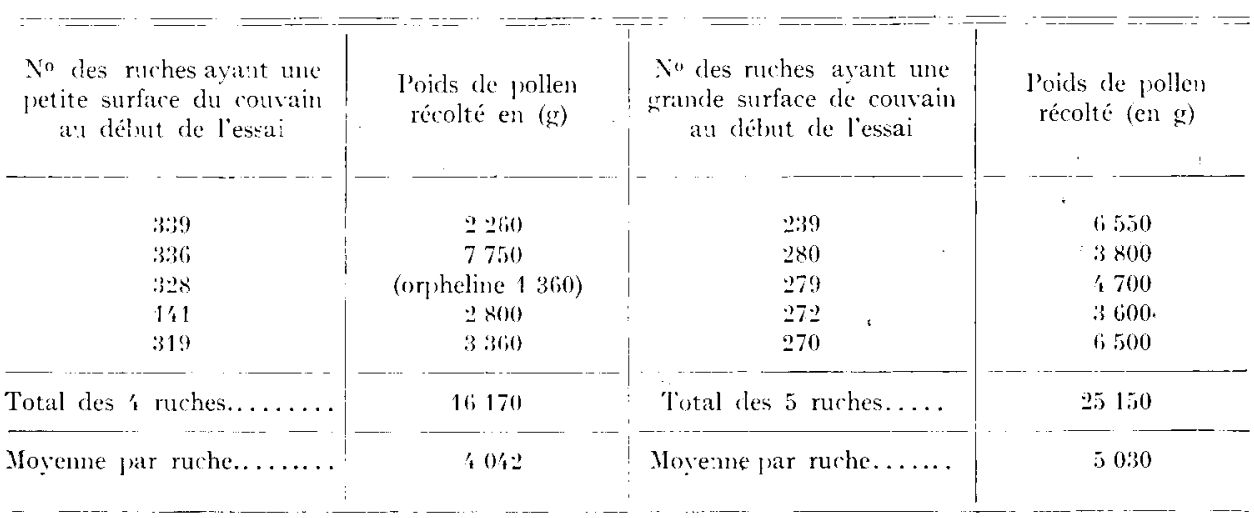

Récolte totale du lot de a ruchos (sans les orphelines) : 11320

Moyenne générale récoltée par l'ensemble du lot : 1531 
passage des butineuses devient de plus en plus réduit. Cependant il faut remarquer que ces résultats sont contredits par ceux obtenus avec les grilles à 6 ou 9 rangées de trous. Dans ce cas, les résultats sont inversés, mais cela prouve que lorsque l'abeille a six rangs d'ouvertures pour passer, la différence avec l'ouverture totale sur neuf rangs est insensible. Il s'ajoute encore à cela d'autres explications en rapport avec le choix des ruches dans les deux lots. Il en sera question au chapitre suivant. Les abeilles ayant eu à traverser trois rangs d'ouvertures ont récolté en moyenne $4829 \mathrm{~g}$ de pollen par ruche. Ce résultat est très intéressant en dépit de la différence de rendsment que l'on constate par rapport aux grilles à un seul rang de passage. En effet,

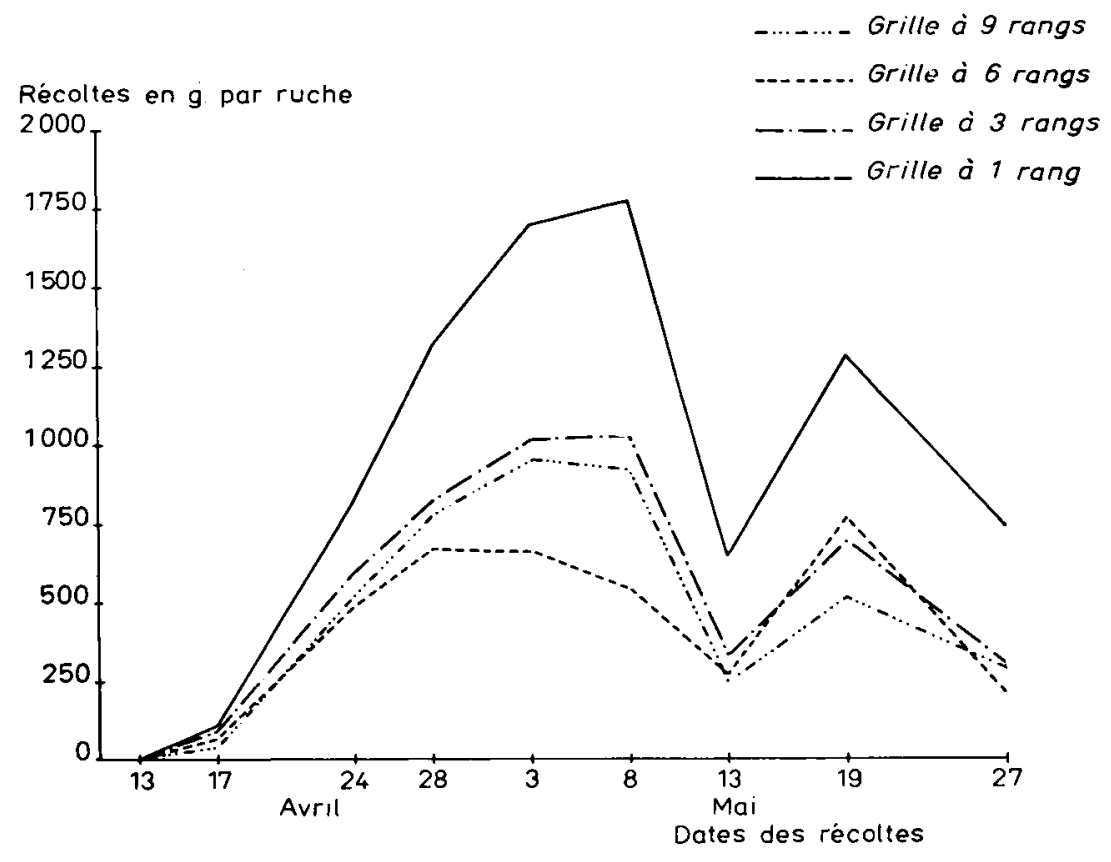

Fic. 2. - Échelonnement des récoltes dans le temps suivant le nombre de rangs libres dans la grille

nous verrons plus loin que ce système n'a apporté aucune pertubation dans le développement des ruches tandis qu'on ne peut en dire autant des grilles où le passage a été réduit au minimum. Le "coefficient de bousculade " a donc bien une influence sur le poids de pollen recueilli par les grilles des pièges à pollen.

\section{Importance d'une ouverture libre complémentaire.}

On peut penser qu'une ruche munie d'une trappe dont la grille laisse un passage réduit doit être gênée au bout d'un certain temps, ne serait-ce que pour la circulation des mâles, l'évacuation des cadavres et des déchets, la ventilation, etc. Pour étudier ce phénomène, nous avons ouvert la sortie normale à la base de la ruche sur la moitié des colonies. Les 20 ruches " ouvertes " à l'arrière et les 20 ruches " fermées " à l'arrière ont été réparties également dans les quatre lots possédant les différentes grilles. Cette expérience doit être légèrement dissociée de l'ensemble du travail. L'ouver- 
ture des 20 colonies a eu lieu au moment de la pleine floraison du ciste. Les résultats obtenus sont portés sur le tableau 5 .

Les résultats globaux montrent, qu'en Io jours, la récolte moyenne des ruches A ayant une ouverture complémentaire ( $\mathrm{I} 628,75 \mathrm{~g}$ ) est supérieure à celle des ruches $\mathrm{B}$ fermées à l'arrière $\left(\mathrm{I}_{3} \mathrm{I} 7,5 \mathrm{~g}\right)$. Cette différence est relativement importante : 3 II, $25 \mathrm{~g}$, soit par ruche et par jour : $3 \mathrm{I}$ g. Si l'on analyse les résultats de plus très, on s'aperçoit que les lots de ruches ayant des trappes à pollen où le passage est très réduit ( $\mathrm{I}$ et 3 rangs de trous) montrent des écarts dans les récoltes beaucoup plus importants entre les groupes A et $B$, que ceux enregistrés sur la moyenne des ruches. A l'inverse, les lots de ruches

\section{TABLFAU 5}

Récolte de pollen par les ruches avec ou sans sortie supplémentaire

\begin{tabular}{|c|c|c|c|c|c|c|c|}
\hline \multirow{2}{*}{$\begin{array}{c}\text { Type de trappe } \\
\text { à pollen }\end{array}$} & \multicolumn{3}{|c|}{$\begin{array}{l}\text { A) Pollen récolté par les ruches } \\
\text { ouvertes à l'arrière (en } g \\
\text { par ruche) }\end{array}$} & \multicolumn{3}{|c|}{$\begin{array}{l}\text { B) Pollen récolté par les ruches } \\
\text { fermées à l'arrière (en g } \\
\text { par ruche) }\end{array}$} & \multirow{2}{*}{$\begin{array}{l}\text { Différence de } \\
\text { récolte entre } \\
\text { A et } B \text { (pendant } \\
10 \text { jours, Jar } \\
\text { ruche) }\end{array}$} \\
\hline & $\begin{array}{ll}\mathrm{du} & 3 / 5 \\
\text { au } & 8 / 5\end{array}$ & $\begin{array}{c}\text { du } 8 / 5 \\
\text { au } 13 / 5\end{array}$ & $\begin{array}{c}\text { Pendant les } \\
10 \text { jours }\end{array}$ & $\begin{array}{ll}\text { dul } & 3 / 5 \\
\text { au } & 8 / 5\end{array}$ & $\begin{array}{cc}\text { du } & 8 / 5 \\
\text { au } & 13 / 5\end{array}$ & $\begin{array}{c}\text { Pendant les } \\
10 \text { jours }\end{array} \mid$ & \\
\hline Grille à 1 rang. ........ & 1940 & 670 & 2610 & 1620 & 600 & 2220 & +390 \\
\hline Grille à 3 rangs $\ldots$. & 1290 & 350 & 1640 & 860 & 290 & 1150 & +490 \\
\hline Grille à 6 rangs $\ldots$ & 725 & 250 & 975 & 437,5 & 300 & 737,5 & $+237,5$ \\
\hline Grille à 9 rangs $\ldots .$. & 1040 & 250 & 1290 & 875 & 287,5 & 1162,5 & $+128,5$ \\
\hline $\begin{array}{l}\text { Moyenne pour les \& types } \\
\text { de trappes à pollen.... }\end{array}$ & 1248,75 & 380 & 1628,75 & 948,12 & 369,37 & 1317,5 & $+311,25$ \\
\hline
\end{tabular}

où le passage dans les trappes est relativement facile (6 et 9 rangs de trous) montrent qu'il y a moins d'écart entre les groupes A et B. Mais dans tous les cas pris individuellement, les récolte; des colonies du groupe $A$ sont toujours supérieures aux récoltes des colonies du groupe B. L'ouverture supplémentaire à l'arrière de la ruche est un facteur favorable à la récolte de pollen puisque le gain obtenu est de $23,62 \mathrm{p}$. Ioo en moyenne. A priori, nous aurions pu penser que les ruches, où les abeilles avajent comme seule sortie la trappe, devaient récolter plus de pollen puisque les autres pouvaient en apporter dans la ruche plus librement en passant par le trou de vol. Or, c'est exactement l'inverse qui se passe et les tiroirs des trappes du groupe A se remplissent plus que ceux du groupe B. Cette ouverture libre permet la sortie des mâles, ce qui évite leur blocage et leur mort dans les grilles qu'ils essaient vainement de traverser. D'autres part, les cadavres et les déchets sont plus facilement évacués à la base de la ruche. Enfin, l'aération de la colonie est meilleure et cette deuxième sortie offerte aux butinueuses évite une congestion trop poussée de la colonie. Une autre remarque peut être faite après examen du tableau 5 . Nous avons donné les chiffres des récoltes du $3 / 5$ au $8 / 5$ d'tune part et du $8 / 5$ au r3/5 d'autre part. Ces 


\section{ILANCIE I}

\section{La trappe à pollen en tosition supérieure}

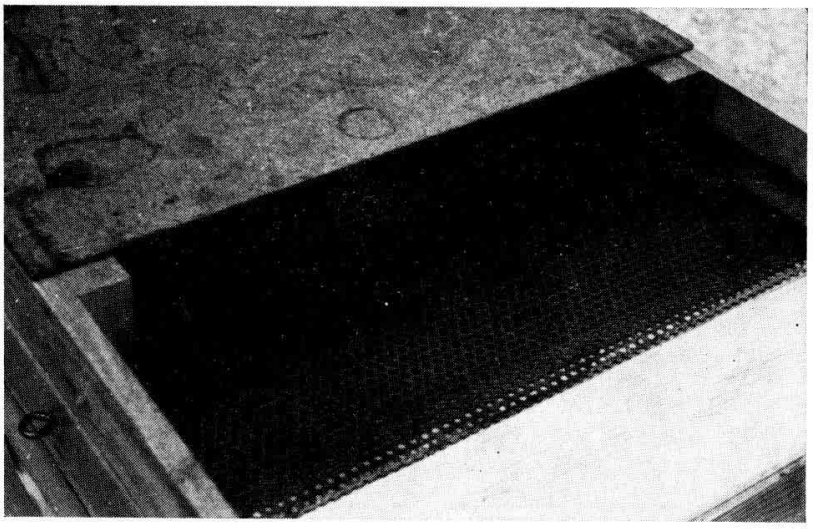

Fic. 3

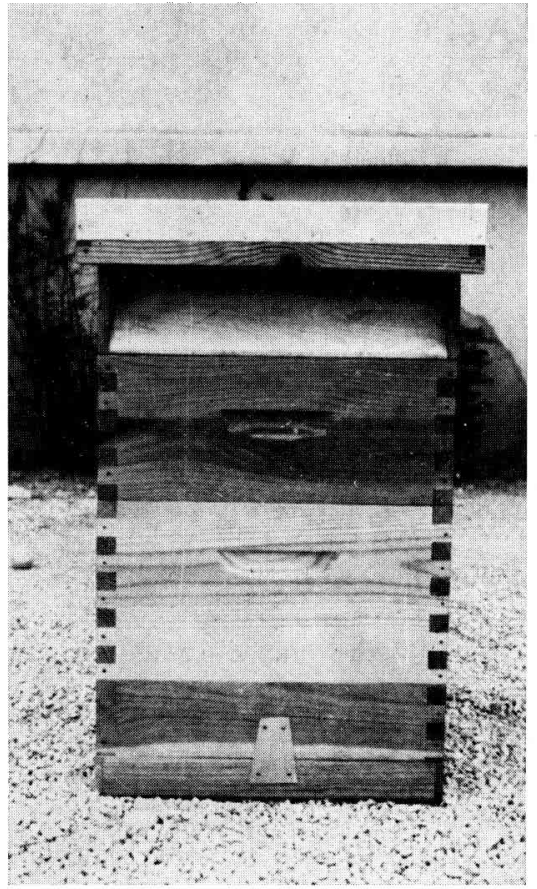

Fis. 4 
Planche in

La trappe à pollen en position supérieure

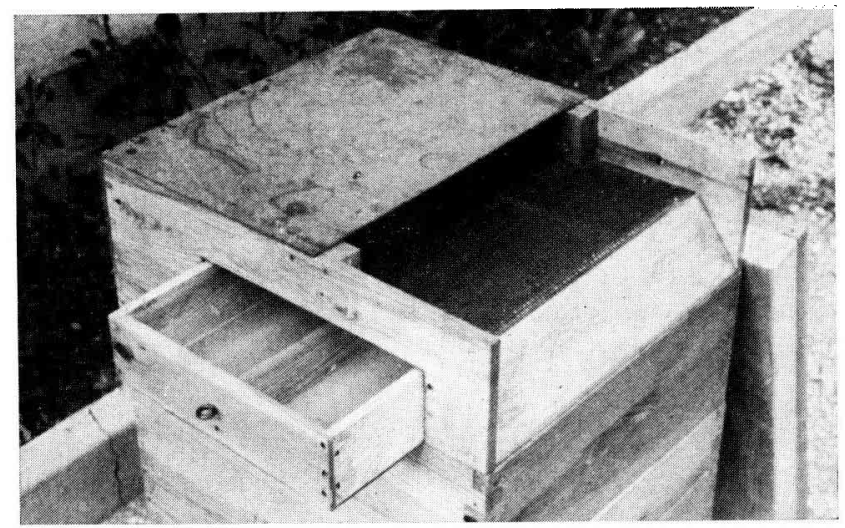

FII. 5

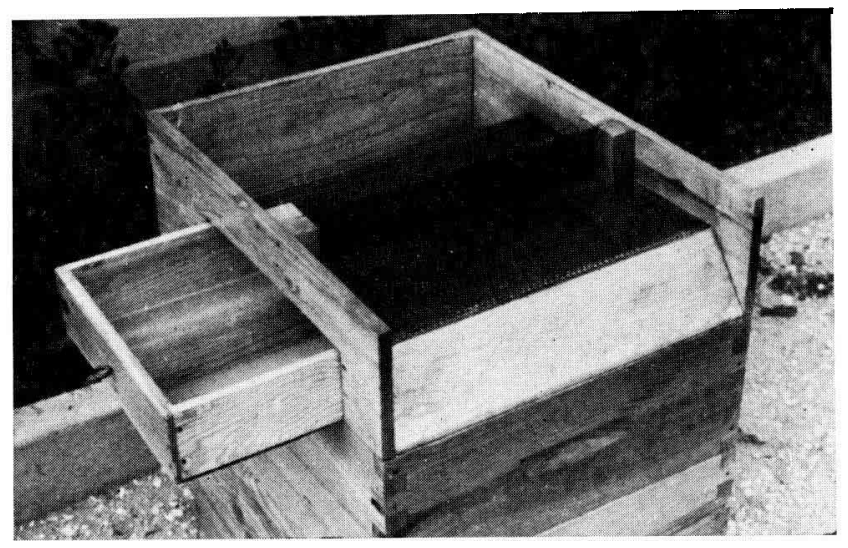

Fig. 6

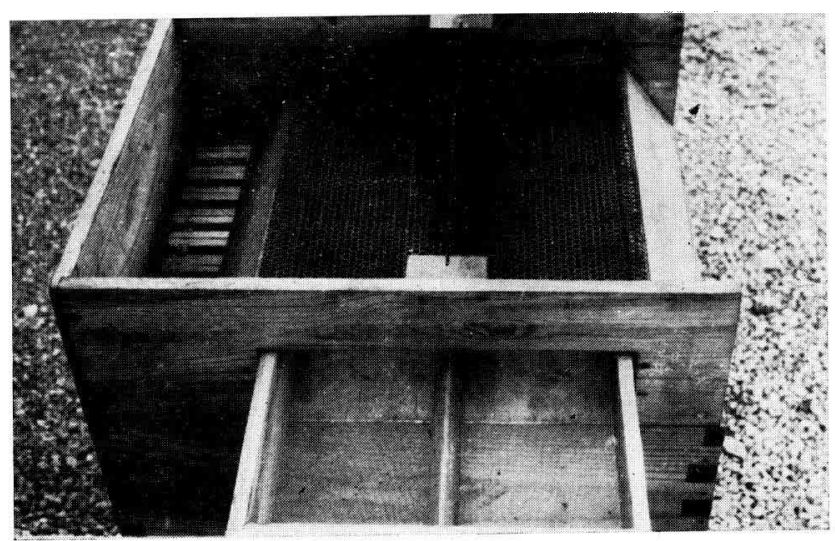

FIC. 7 
deux périodes sont totalement différentes, la première correspond à la période la plus favorable de la pleine floraison des cistes, tandis que la seconde correspond à une période de très mauvais temps (fig. I) avec des récoltes plus faibles. Nous constatons que les écarts entre les groupes A et B sont importants lors de gros apports de pollen (du $3 / 5$ au $8 / 5$ ) et presque nuls par mauvais temps (du $8 / 5$ au $13 / 5$ ). Dans cette deuxième période, les résultats sont parfois inversés, le groupe $B$ récoltant plus que le groupe $\mathrm{A}$ (ruches à 6 et à 9 rangs de trous.) Dans ce dernier cas, on peut supposer que les abeilles ayant trop de surface de passage s'occupent principalement à calfeutrer les ouvertures pour éviter le refroidissement du couvain. On peut noter également que les colonies du groupe $\mathrm{A}$ ont eu une chute de récolte assez importante lors de la période de mistral (du8/5 au I3/5), tandis que celles du groupe B ont accusé une chute proportionnellement beaucoup plus faible.

$\left.3^{\circ}\right)$ Récolte $d u$ pollen en fonction du développement de la colonie d'abeilles et de la surface de couvain au début de l'expérience

L'influence de la surface de couvain sur la récolte du pollen a déjà été signalée par Louveaux (I958). Nous avons complété et confirmé ses résultats sur un grand nombre de ruches et dans une région différente. Pour cette étude nous avons considéré nos colonies séparées en deux lots, celle; dont les surfaces de couvain sont les

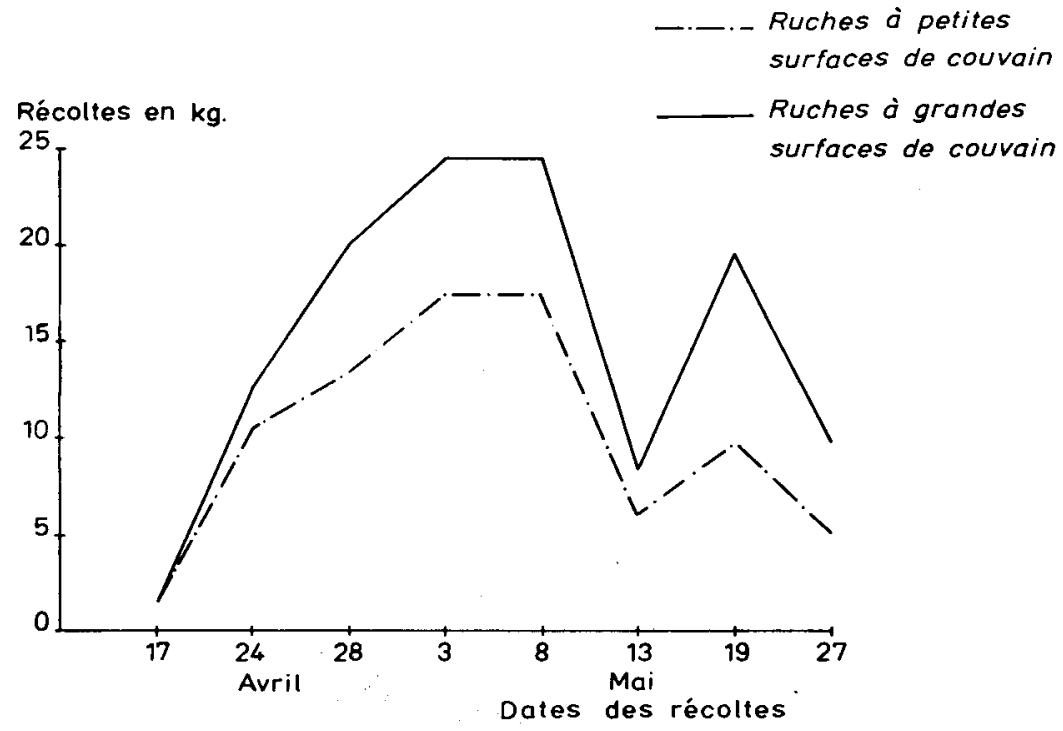

FIG. 8. - Pollen récolté en fonction du temps et de la surface de couvain

plus faibles et celles dont les surfaces de couvain sont les plus grandes. Ces surfaces de couvain sont mesurées avant l'expérience et les colonies devenues orphelines en cours d'essai ne sont pas prises en considération. Nous avions 37 ruches en expérience dont la moyenne de surface de couvain était de $I_{59,6}$ points (soit $63,84 \mathrm{dm}^{2}$ ). La colonie la plus faible possédait go points et la plus forte 225 points. Dans tout le lot. 3 ruches seulement avaient moins de roo points de couvain et 6 ruches avaient plus 
de 200 points. Les colonies ont été réparties aussi équitablement que possible dans les lots ayant des trappes à grilles différentes. Cependant le lot de ruches, dont les grilles comportent six rangées de trous, sont plus homogènes au point de vue couvain, ce qui fait qu'il est plus difficile de séparer " grandes » et " petites » surfaces. Dans chaque lot, nous avons considéré cinq ruches à couvain étendu et cinq ruches à couvain plus réduits. La figure 8 donne la récolte réalisée au cours du temps par les colonies à grande et à petite surface de couvain. Les différences sont principalement nettes lors de la pleine floraison et des gros apports de pollen et elles sont atténuées si la récolte est faible. En résumé pendant 40 jours :

- les I8 ruches à surface de couvain faible ont récolté 8 I $490 \mathrm{~g}$, soit $4527 \mathrm{~g}$ de moyenne par ruche.

- les rg ruches à grande surface de couvain ont récolté I I9 $440 \mathrm{~g}$, soit $6286 \mathrm{~g}$ de moyenne par ruche.

Cette différence est très significative : les ruches ayant peu de couvain récoltent moins de pollen que les autres.

TABLEAU 6

Récolle de pollen en fonction de la surface du couvain

\begin{tabular}{|c|c|c|c|c|c|c|}
\hline \multirow[b]{2}{*}{ Type de trappe à pollen } & \multirow{2}{*}{$\begin{array}{c}\text { Surface } \\
\text { moyenne du } \\
\text { couvain par } \\
\text { ruche (en } \\
\text { points) }\end{array}$} & \multicolumn{5}{|c|}{ Moyennes des récoltes de pollen (en g) } \\
\hline & & $\begin{array}{l}\text { Récolte sur } \\
\text { tout le lot }\end{array}$ & $\begin{array}{l}\text { Récolte des } \\
\text { colonies à } \\
\text { couvain dé- } \\
\text { veloppé }\end{array}$ & $\begin{array}{c}\text { Récolte des } \\
\text { colonies à } \\
\text { couvain faible }\end{array}$ & $\begin{array}{c}\text { Récolte des } \\
\text { deux colonies } \\
\text { les plus fortes }\end{array}$ & $\begin{array}{l}\text { Récolte des } \\
\text { deux colonies } \\
\text { les plus } \\
\text { faibles }\end{array}$ \\
\hline Grille à 1 rang & 165,5 & 8355 & 9940 & 6770 & 8775 & 6625 \\
\hline Grille à 3 rangs........ & 152,1 & 4829 & $61.7 \%$ & $3{ }^{\prime} 8^{\prime}$ & 7475 & 3030 \\
\hline Grille à 6 rangs........ & 147,6 & 3471 & 3430 & 3512 & 4085 & 3400 \\
\hline Grille à 9 rangs........ & 171,5 & 4591 & 5030 & 4042 & 5150 & 2530 \\
\hline $\begin{array}{l}\text { Ensemble de toutes les } \\
\text { ruches } \quad \ldots \ldots \ldots \ldots \ldots . .\end{array}$ & 159,6 & 5430 & 6286 & 4527 & & \\
\hline
\end{tabular}

Le tableau 6 apporte des détails supplémentaires sur les apports de pollen des ruches faibles et des ruches fortes. On y a porté en particulier les récoltes effectuées par les colonies les plus populeuses et par les colonies les plus faibles. Cette dernière comparaison montre que la récolte peut être le double dans les ruches les plus fortes par rapport aux plus faibles. Même si l'on considère le lot comportant des trappes à grilles à 6 rangs, et dont les variations de l'étendue du couvain sont faibles, on s'aperçoit que si l'on prend les deux colonies les meilleures (totalisant 364 points de couvain) et les deux colonies les plus faibles (totalisant 223 points de couvain) la différence de moyenne de récolte attteint $685 \mathrm{~g}$. 
La corrélation entre l'importance du développement du couvain et la récolte du pollen permet d'interpréter plus facilement les anomalies que nous avons signalées plus haut. En effet, nous avions trouvé que les colonies munies de trappes à 6 rangs récoltaient moins de pollen que celles équipées de trappes à 9 rangs. L'examen du tableau 6 de la surface moyenne du couvain par ruche dans chaque lot permet de comprendre l'inversion des résultats. Les ruches "à 9 rangs " avaient I7 I,5 points de couvain alors que les ruches "à 6 rangs " en avaient seulement I47,6. Il est donc normal que les premières aient récolté beaucoup plus de pollen malgré un passage plus libre pour les butineuses. De même les ruches "à 3 rangs " auraient récolté beaucoup plus de pollen par rapport aux ruches à " 9 rangs " si elles avaient eu une surface moyenne de couvain supérieure à I52, I points. Cet ensemble de considérations complémentaires permet d'insister à nouveau sur l'intérêt de la trappe à pollen possédant 3 rangées de trous pour le passage des abeilles.

\section{Cas des ruches orphelines.}

Au cours de nos essais, trois ruches sont devenues orphelines : deux ruches ayant des trappes à 6 rangs de trous et une ruche ayant 9 rangs de trous. Dans le premier cas, seule la ruche $n^{0}$ I 26 a survécu pendant l'expérience, la ruche $n^{0} 277$ ayant disparu très vite. Dans le deuxième cas, la ruche $n^{\circ} 328$ a permis également de faire des récoltes et des relevés. La figure 9 donne les courbes de récolte de ces deux colonies

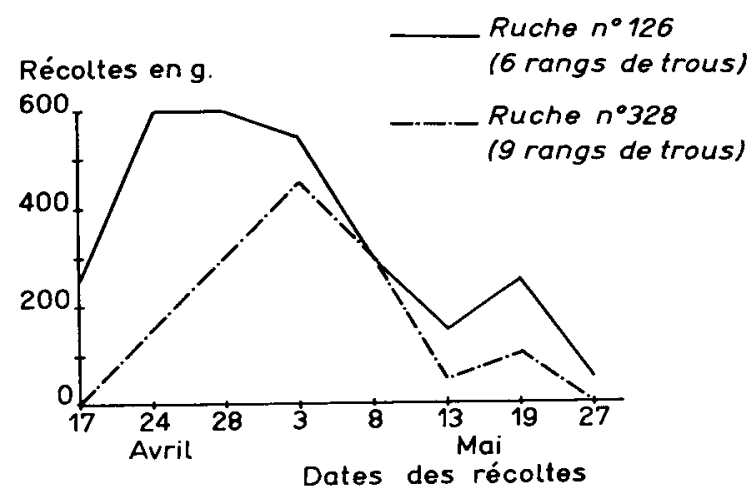

FIG. 9. - Pollen récolté par les ruches orphelines.

d'abeilles en fonction du temps. On remarque tout d'abord que les deux ruches ont une récolte moyenne très faible : $2055 \mathrm{~g}$ par colonie en 40 jours, soit $2750 \mathrm{~g}$ pour la ruche 126 (alors que la récolte moyenne de son groupe est de $347 \mathrm{I} \mathrm{g}$ ) et I $360 \mathrm{~g}$ pour la ruche 328 (alors que la récolte moyenne de son groupe est de $459 \mathrm{I} \mathrm{g}$ ).

Même si l'on prend dans chacun de ces groupes (tabl. 6) la récolte moyenne des deux ruches à surface de couvain très réduite, la récolte des abeilles orphelines est bien inférieure. D'autre part si l'on compare la figure 9 à la figure 2 , on remarque que les récoltes de pollen entre les orphelines et les ruches normales des mêmes lots subissent sensiblement les mêmes accidents au cours du temps notamment la chute dans les récoltes du $\mathrm{I}_{3} / 5$ et du $27 / 5$ et la remontée du I9/5. La ruche $\mathrm{n}^{0}$ I 26 a perdu $2 \mathrm{~kg}$ au cours de $1^{\prime}$ expérience et la ruche $n^{\circ} 328$ n'a pas varié de poids. Au début de la pose des trappes, la ruche $n^{0} \mathrm{I} 26$ a récolté beaucoup plus que la ruche $n^{0} 328$ mais les surfaces de 
couvain avant 1'essai étaient respectivement de I 68 points et de I02 points, ce qui explique cette différence. La ruche $\mathrm{n}^{0}$ I 26 devait être tout à fait normale les Io premiers jours car elle a récolté pendant cette période plus que la moyenne des ruches de son lot. La ruche $n^{0} 328$ par contre a toujours été une colonie à récolte faible, bien que n'étant pas orpheline au début. On peut supposer que la tuche $n^{0} 328$ est devenue normalement orpheline, les abeilles se disposant à remplacer la reine dont le couvain était médiocre ( 102 points) tandis que la ruche no $n^{\circ} 6$ serait devenue orpheline accidentellement.

En résumé, l'importance du couvain dans une ruche régit bien la récolte du pollen par les abeilles. Les ruches ayant une grande surface de couvain en ont récolté $6286 \mathrm{~g}$ les rûches ayant une petite surface de couvain en ont récolté $4527 \mathrm{~g}$. et les ruches orphelines seulement $2055 \mathrm{~g}$.

\section{$\left.4^{\circ}\right)$ Infuence de la récolte du pollen sur le développement du couvain et sur le poids des ruches}

Nous avons tenté au cours de ces essais de savoir quelle était l'influence réelle du prélèvement de pollen pratiqué sur les colonies d'abeilles. Cette question a été souvent discutée, mais en fait il n'existe aucun travail sur la question faisant état de mesures précises sur un nombre de ruches suffisant, notamment en ce qui concerne les surfaces de couvain et le poids des ruches, avant et après les essais. Une expérimentation est également en cours pour connaître l'influence du prélèvement de pollen sur les récoltes ultérieures de miel au cours de la même saison. Elle fera l'objet d'une publication après deux années d'essais.

En ce qui concerne l'influence de la récolte du pollen sur le développement du couvain pendant la pose des trappes, les résultats sont consignés dans le tableau 7 . Nous remarquerons d'abord que la surface de couvain a augmenté dans tous les lots sauf pour les ruches possédant des trappes à pollen avec des grilles à I rang de trous ( -8 points). Les ruches dont la surface de couvain a pris le plus d'ampleur sont celles possédant des grilles à 3 rangs de trous, leur couvain ayant augmenté de 28,9 points par ruche pendant l'opération (soit II,56 $\mathrm{dm}^{2}$ ). En moyenne sur les 37 ruches le couvain s'est développé de Io,3 points par ruche, ce qui est loin d'être négligeable. On ne peut donc pas dire que des trappes à pollen placées sur des ruches empêchent le développement de la colonie, sauf dans les cas limites (trappes avec très faible passage). 'Toutefois, si l'on considère les choses d'un peu plus près on s'aperçoit que les colonies ayant le plus de couvain au début de l'expérience ont eu tendance à voir ce couvain régresser ou tout au moins rester stationnaire. Par contre, les colonies ayant moins de couvain à l'origine se sont bien développées en général, et ce couvain a augmenté dans de bonnes proportions au cours de l'essai, même celui des ruches avec grilles à pollen à un rang de passage (gain I06 points). Les très fortes populations sont perturbées par les trappes à pollen. Lin effet, l'aération de la ruche et la circulation des abeilles se trouvent alors très réduites et ceci est particulièrement net dans le lot des grilles à I rang de trous de passage. Dans cet exemple, la perte est de $I 8 \mathrm{I}$ points de couvain contre une perte de I4 points seulement pour les colonies fortes ayant des grilles à 9 rangs de trous, laiss ınt un passage beaucoup plus libre. Ia ruche qui a vu son couvain diminuer le plus est la ruche $n^{0} 368$ (lot des grilles à I rang de trous). Sa surface de couvain est passée de 2 I 4 points à II 7 points soit une perte de 97 points et 
également une perte de $8 \mathrm{~kg}$. Par contre, la ruche $\mathrm{n}^{0}$ I I 2 (lot des grilles à 3 rangs de trous) a vu_sa surface de couvain augmenter de Ioo à 243 points soit un gain de r43:points, son poids par contre n'a pas varié. Si l'on considère l'ouverture supplémentaire à l'arrière de la ruche, on s'aperçoit que celle-ci n'a eu aucune influence sur

\section{TABLEAU 7}

Influence de la récolte du pollen sur le développement du couvain (en points)

\begin{tabular}{|c|c|c|c|c|c|c|}
\hline \multirow{2}{*}{ Type de trappe à pollen } & \multirow{2}{*}{$\begin{array}{l}\text { Groupe à forte (C) } \\
\text { ou à faible (c) } \\
\text { surface de couvain }\end{array}$} & \multirow{2}{*}{$\begin{array}{l}\text { Nombre } \\
\text { de ruches }\end{array}$} & \multicolumn{3}{|c|}{ Surface totale du couvain } & \multirow{2}{*}{$\begin{array}{l}\text { Perte ou } \\
\text { gain moyen } \\
\text { par ruche }\end{array}$} \\
\hline & & & $\begin{array}{l}\text { Avant } \\
\text { récolte }\end{array}$ & $\begin{array}{l}\text { Après } \\
\text { récolte }\end{array}$ & $\begin{array}{l}\text { gain ou } \\
\text { perte }\end{array}$ & \\
\hline \multirow{2}{*}{ Grille à 1 rang } & $\mathrm{C}$ & 5 & 1015 & $83{ }^{\prime}$ & -181 & \multirow{2}{*}{-8} \\
\hline & $c$ & 5 & 645 & 751 & +106 & \\
\hline \multirow{2}{*}{ Grille à 3 rangs } & C & 5 & 965 & 933 & -32 & \multirow{2}{*}{$+28,9$} \\
\hline & $c$ & 5 & 556 & 877 & +321 & \\
\hline \multirow{2}{*}{ Grille à 6 rangs } & $\mathrm{C}$ & 4 & 67 ' & 711 & $+\quad 37$ & \multirow{2}{*}{$+14,3$} \\
\hline & $c$ & 4 & 507 & 588 & +81 & \\
\hline \multirow{2}{*}{ Grille à 9 rangs } & $\mathrm{C}$ & 5 & 1016 & 1002 & $-11^{\prime}$ & \multirow{2}{*}{+6} \\
\hline & $c$ & 隹 & 528 & 592 & $+6 \mathbf{k}$ & \\
\hline Total des lots des ruches & $\mathrm{C}+c$ & 37 & 5906 & 6288 & +382 & $+10,3$ \\
\hline
\end{tabular}

le développement du couvain: les ruches "fermées » ou " ouvertes " à l'arrière se comportent de la même manière à l'intérieur de chacun de leur lot. Il est intéressant de signaler que dans toutes ces ruches, on a retiré par la suite pour éviter des essaimages naturels 3 paquets d'abeilles de I $\mathrm{kg}$ (ruches $\mathrm{n}^{0} 276,265$ et 3 I9) et deux cadres de couvain à la ruche $\mathrm{n}^{0}$ II 2 .

La récolte du pollen a très peu infué sur le poids des ruches pendant les 40 jours de pose des trappes. Il faut tenir compte du fait que la miellée a été quasi nulle pendant toute la période incriminée. Les résultats sont portés sur le tableau 8 . Seules les colonies ayant des trappes à I rang de passage libre ont perdu du poids et principalement les colonies les plus lourdes et ayant le plus de couvain. Toutes les autres ruches sont bénéficiaires, mais l'augmentation moyenne par ruche est négligeable. Le lot des trappes à pollen à 3 rangs de passage libre possède les ruches qui ont pris le plus de poids (moyenne par ruche $+\mathrm{I}, 65 \mathrm{~kg}$ ). Sur l'ensemble des 37 ruches l'augmentation moyenne a été de $0,08 \mathrm{I} \mathrm{kg}$. L'ouverture supplémentaire n'a pas influé sur le poids des ruches. C'est la ruche $n^{0}$ I54 (grille à 6 rangs de trous) qui a perdu le plus de poids en 40 jours : II $\mathrm{kg}$. Par contre, c'est la ruche $\mathrm{n}^{\circ}$ 3I 5 (dans le même lot) qui a augmenté le plus : $6,5 \mathrm{~kg}$. Le poids moyen des ruches au moment de la mise en hiver- 
nage était très satisfaisant comme le montre le tableau 8 . Avant l'essai, le poids moyen des ruches était de $26,3 \mathrm{~kg}$; à 1'hivernage, il était de $3 \mathrm{I}, 07 \mathrm{~kg}$ soit un gain moyen de $4,77 \mathrm{~kg}$. Là encore, on s'aperçoit que le lot des trappes à 3 rangées de trous est bénéficiaire par rapport aux autres $(32,28 \mathrm{~kg}$ de moyenne par ruche à l'hivernage).

\section{TABLEAU 8}

Influence de la récolte du pollen sur le poids des ruches (en $\mathrm{kg}$ )

\begin{tabular}{|c|c|c|c|c|c|c|c|}
\hline \multirow{2}{*}{ Type de trappe à pollen } & \multirow{2}{*}{$\begin{array}{l}\text { Groupe à forte } \\
\text { (C) ou à faible } \\
\text { (c) surface de } \\
\text { couvain }\end{array}$} & \multirow{2}{*}{$\begin{array}{l}\text { Nombre } \\
\text { de ruches }\end{array}$} & \multicolumn{3}{|c|}{ Poirls total des ruches } & \multirow{2}{*}{$\begin{array}{l}\text { Perte ou } \\
\text { gain moyen } \\
\text { par ruche }\end{array}$} & \multirow{2}{*}{$\begin{array}{l}\text { Poids moyen } \\
\text { des ruches a } \\
\text { l'hivernage }\end{array}$} \\
\hline & & & $\begin{array}{l}\text { Avant } \\
\text { récolte }\end{array}$ & $\begin{array}{l}\text { Après } \\
\text { récolte }\end{array}$ & $\begin{array}{l}\text { Gain ou } \\
\text { perte }\end{array}$ & & \\
\hline \multirow{2}{*}{ Grille à 1 rang } & C & 5 & 152 & 130,5 & $-21,5$ & \multirow{2}{*}{$-2,35$} & \multirow{2}{*}{31,16} \\
\hline & $c$ & 5 & 123 & 120,5 & $-\quad 2,5$ & & \\
\hline \multirow{2}{*}{ Grille à 3 rangs } & C & 5 & 136 & 142 & +6 & \multirow{2}{*}{$+1,65$} & \multirow{2}{*}{32,28} \\
\hline & $c$ & 5 & $12 \underline{4}$ & 134,5 & $+10,5$ & & \\
\hline \multirow{2}{*}{ Grille à 6 rangs } & C & 4 & 110 & 111,5 & $+1,5$ & \multirow{2}{*}{$+0,31$} & \multirow{2}{*}{30} \\
\hline & $\epsilon$ & 4 & 98,5 & 99,5 & +1 & & \\
\hline \multirow{2}{*}{ Grille à 9 rangs } & C & 5 & 133,5 & 139,5 & +6 & \multirow{2}{*}{$+0,88$} & \multirow{2}{*}{30,87} \\
\hline & $c$ & 4 & 99 & 101 & +2 & & \\
\hline Total des lots de ruches & $\mathrm{C}+c$ & 37 & 976 & 979 & +3 & $+0,081$ & 31,07 \\
\hline
\end{tabular}

$\left.5^{\circ}\right)$ Remarques diverses.

a) Choix dans la récolte par les colonies.

Nous avons déjà signalé que 60 p. Ioo de la récolte du pollen étaient constitués par le pollen de ciste. Cependant, ce pourcentage a varié d'une ruche à l'autre et certaines colonies ont récolté penđant la période de pleine floraison du pollen de ciste presque pur (9o à $95 \mathrm{p}$. IOO). Le coquelicot (Papaver rhaeas) également a intéressé très diversement les abeilles. Certaines ruches l'ont ignoré complètement, d'autres l'ont découvert tôt ou tard (ro jours d'écart). Enfin, certaines ruches en ont amassé beaucoup alors que la moyenne est de $2 \mathrm{p}$. Ioo dans le pollen récolté. Il en est de même pour les autres pollens qui ont intéressé diversement les colonies d'abeilles.

b) Ruches à fortes récoltes et ruches à faible récolte.

Si l'on reprend les tableaux I, 2,3 et 4 , on se rend compte que certaines ruches ont fait des récoltes de pollen se situant en dehors des chiffres moyens. On s'aperçoit que 3 ruches ont fourni plus de $\mathrm{Io} \mathrm{kg}$ de pollen.

La ruche $\mathrm{n}^{\circ}$ I59 a récolté $\mathrm{I} 3$, I $\mathrm{kg}$ : elle a perdu 35 points de couvain

la ruche $\mathrm{n}^{\circ} \mathrm{I} 42$ a récolté $\mathrm{I} 2,8 \mathrm{~kg}$ : elle a perdu 23 points de couvain

la ruche $\mathrm{n}^{\circ} 276$ a récolté $\mathrm{II}, 2 \mathrm{~kg}$ : elle a perdu 44 points de couvain.

Annales de l'Abeille. - i 963 . 
Mais cette dernière fournira au cours de la saison I $\mathrm{kg}$ d'abeilles et pèsera à 1'hivernage $3 \mathrm{I} \mathrm{kg}$ contre $28 \mathrm{~kg}$ au début de l'essai.

Dans ce même groupe (tabl. $x$ ), on peut remarquer que la ruche $n^{\circ} 368$ n'a récolté que $4,750 \mathrm{~kg}$ et cependant elle possédait au début des essais 214 points de couvain et pesait 3I $\mathrm{kg}$. Non seulement sa récolte est faible mais elle a perdu $6 \mathrm{~kg}$ et 97 points de couvain en 40 jours. Nous enregistrons ces résultats sans pouvoir leur apporter une explication. Cette ruche est cependant maintenue en bon état ensuite: à 1 'hivernage elle pesait $35 \mathrm{~kg}$.

De même dans le tableau 2, la différence de récolte entre la ruche $n^{0} 273(8,3 \mathrm{~kg})$ et la ruche $\mathrm{n}^{0}$ Io8 $(2,4 \mathrm{~kg})$ ne s'explique pas. Ce sont deux ruches de poids et de surface de couvain semblables à l'origine. D'autres exemples se retrouvent dans les tableaux 3 , et 4 la ruche $n^{\circ} 336$ (faible couvain) récolte $7,75^{\circ} \mathrm{kg}$ de pollen et elle pèsera $36 \mathrm{~kg}$ à $1^{\prime}$ hivernage.

L'âge des reines serait peut-être à considérer, mais nos renseignements à ce sujet sont incomplets. Cependant, les trois ruches ayant récolté le plus de pollen possédaient des reines de deux ans.

\section{c) Augmentation de la récolte au cours de la floraison du ciste.}

On peut dire que presque toutes les colonies ont récolté de plus en plus de pollen au fur et à mesure de la floraison du ciste. Seuls les facteurs météorologiques limitants ont diminué ces apports, mais après le rétablissement du beau temps la récolte a repris jusqu'à la fin de floraison. Cependant certaines ruches ont vu leurs récoltes baisser avant la pleine floraison. Par exemple, la ruche $n^{\circ}$ I05 (grille à 6 rangs de trous) a récolté le poids de pollen maximum entre le 24 avril et le 28 avril. Ensuite, sa récolte baisse régulièrement malgré la pleine floraison. Cependant, on constate à la fin qu'elle n'a pas été affaiblie par la pose de la trappe à pollen.

Autre remarque : dans le même lot, certaines ruches récoltent très vite du pollen et $1^{\prime}$ on peut voir par exemple la ruche $\mathrm{n}^{\circ} 34$, récolter $500 \mathrm{~g}$ dans les 4 premiers jours alors que la ruche voisine, $\mathrm{n}^{\circ} 278$, ayant un couvain un peu plus étendu ne récolte rien dans le même laps de temps. Cependant la récolte totale des deux ruches diffère très peu (tab1. r).

\section{d) Période de récolte maximum.}

La période maximum de la récolte de pollen se situe entre le 28 avril et le 8 mai. C'est entre le 5 et 8 mai que les ruches fortes ont récolté le plus de pollen sauf les ruches à grilles ayant 6 et 9 rangs de trous qui ont montré une très légère baisse de rendement. Pendant ces 5 jours, la colonie no $\mathrm{I}_{42}$ a récolté $2900 \mathrm{~g}$ de pollen soit $580 \mathrm{~g}$ de moyenne par jour. Mais dans la période précédente, entre le 24 et 28 avril, la ruche champion no $\mathrm{I} 59$ en a récolté $235^{\circ} \mathrm{g}$ soit $587 \mathrm{~g}$ par jour. Ces chiffres se passent de tous commentaires. Si l'on prend l'ensemble des ruches, on peut calculer qu'il a été récolté $83,5 \mathrm{~kg}$ de pollen entre le 28 avril et le 8 mai pendant les Io jours de pleine floraison et par temps favorable, c'est-à-dire $40 \mathrm{p}$. Ioo de la récolte totale effectuée pendant 40 jours. 


\section{III. - DISCUSSION DES RÉSULTATS}

\section{$\mathrm{r}^{\mathrm{o})}$ Récolte du pollen en fonction de la surface de passage pour les butineuses.}

Nous avons montré que les trappes à pollen à 3 rangs de trous pour le passage des abeilles doivent être considérées comme le matériel optimum à utiliser. En effet, seule cette trappe permet une récolte de pollen importante tout en respectant le développement normal de la colonie. Les résultats indiquent dans ce lot de ruches un gain de surface de couvain et un gain de poids au cours de l'expérience. Dans ce même lot, il n'y a pas eu d'orphelinage, ni de mortalité anormale. Deux des ruches ont même donné des essaims au cours de la saison et à 1'hivernage le poids de toutes ces ruches était satisfaisant (minimum $29 \mathrm{~kg}$ ). Par ailleurs, si on considère le tableau 6 , on remarque que la surface moyenne du couvain par ruche dans ce lot est de $I_{5} 2$, I points c'est-à-dire une surface moindre que celle des autres lots, excepté les ruches équipées de grilles à 6 rangs. On peut supposer valablement que si cette surface avait été aussi importante que celle des autres lots, la récolte aurait dû être encore supérieure. Nous avons vu en effet que la surface du couvain est un facteur très important dans la récolte du pollen et que l'inversion qui existe dans les résultats obtenus avec les trappes à 6 et à 9 rangs de passage s'explique facilement si l'on compare les surfaces moyennes de couvain de ces deux lots de ruches. Quant aux trappes avec I rang de passage, le rendement en pollen est spectaculaire mais les ruches souffrent beaucoup plus de ce traitement puisque les colonies perdent du poids et qu'elles voient leur couvain se réduire au cours des esais. Il ne semble donc pas intéressant de les employer dans la pratique, mais ces résultats montrent bien que CHAUvin et LouVEAUX (1956) avaient vu juste en remarquant que le facteur «bousculade», à 1'entrée d'une trappe à pollen, augmentait la récolte dans de grandes proportions. Plus la surface d'entrée des butineuses est restreinte et plus la récolte de pollen est considérable. HIRSCHFELDER (I95I) avait montré de son côté que les butineuses pouvaient être gênées fortement ou pas du tout lors du passage dans la trappe à pollen et cela en fonction du modèle de grille et de la force de la colonie. CHAUvin, dès I955, préconise la tôle perforée pour équiper les trappes à pollen. Celles-ci sont plus régulières que les grillages calibrés, ne se déforment pas et ne nuisent pas aux colonies d'abeilles. Depuis I958, nous récoltons du pollen au moyen des tôles perforées sans le moindre ennui à la Station expérimentale d'Apiculture.

En ce qui concerne l'ouverture supplémentaire à l'arrière de la ruche, CHauvin (I955) la préconisait pour la sortie des mâles et éventuellement des reines vierges ainsi que pour l'évacuation des déchets. Nous avons montré que non seulement cette sortie libre à 1'arrière de la ruche avait les avantages déjà énumérés (aération, vol des mâles, évacuation des cadavres, etc.) mais que les ruches disposant d'une telle sortie ont récolté plus de pollen que les ruches fermées à l'arrière et ceci quel que soit le modèle de grille adopté. Plus les colonies sont peuplées et plus le passage dans la grille de la trappe est réduit, plus l'influence de l'ouverture supplémentaire est nette sur la quantité de pollen récolté. 


\section{0) Récolte du pollen en fonction du développenent du convain}

Louveaux (I950) a montré que l'introduction de couvain ouvert ou de couvain operculé dans une ruche induit une augmentation de récolte de pollen par les butineuses. La présence du couvain est donc un facteur important du déterminisme de la récolte. Ce même auteur ( $95^{8}$ ) écrit que la quantité de pollen récoltée est pratiquement proportionnelle à l'étendue du couvain. Cependant il n'existait pas à l'époque d'appareil satisfaisant pour pratiquer de nombreuses mesures de surface de couvain sans perturber les colonies. C'est maintenant chose faite et nous venons de vérifier cette relation existant entre la surface de couvain et l'importance de la récolte sur 40 ruches.

En ce qui concerne les ruches orphelines,nous avons retrouvé les mêmes résultats que Louveaux (1950). Les ruches orphelines continuent à récolter du pollen d'une façon désordonnée, mais en quantité bien plus faible que les ruches normales.

\section{$\left.3^{\circ}\right)$ Influence des facteurs météorologiques sur la récolte du pollen}

Chauvin (I955) a montré l'importance des facteurs météorologiques sur le rendement des trappes à pollen. Il précise que par temps froid les abeilles confectionnent des pelotes plus petites et que ces dernières sont moins bien retenues dans les tôle perforées servant de pièges. Louveaux (I954 et $195^{8}$ ) précise l'intérêt de ces facteurs. Il pense que ceux-ci influent plus sur les abeilles que sur la flore, bien que cela soit difficile à déterminer. Pour ce dernier auteur, il n'y a plus de récolte en dessous de + Io $^{\circ}$. Nous avons remarqué, dans le Gard, l'importance du "mistral " lorsque celui-ci souffle avec violence pendant plusieurs jours consécutifs. Les auteurs précédents n'ont pas mentionné le vent comme facteut limitant, sans doute parce que les vents enregistrés sont loin de ressembler au mistral. La figure I montre l'influence très marquée de ce vent dans le Sud-Iist, sur la récolte du pollen. Nous pensons d'autre part que les fleurs de ciste sont assez fragiles et que le vent perturbe alors dans ce cas autant la floraison que la sortie des butineuses. Quoi qu'il en soit, la quantité de pollen récoltée dans les périodes très ventées diminue dans de grandes proportions, bien que la température ne devienne pas toujours un facteur limitant. D'autre part, la pluie diurne peut gêner la récolte, si elle est abondante et continue, mais la pluie nocturne ne dérange pas la floraison, les fleurs dı Ciste ne s'épanouissant qu'au cours de la matinée pour faner avant le soir.

\section{$\left.4^{\circ}\right)$ Choix far les colonies dans la récolte du pollen ot quantités récoltées}

Chaque colonie récolte les pollens strivant un choix qui lui est propre et en fonction de la flore mise à sa disposition. Mais cependant, l'abeille s'intéresse presque exclusivement aux plantes communes et comme l'a signalé LovvEAux (I954) une faible partie de la flore est utilisée. Nous avons vu que le spectre des pollens que nous avons récoltés est pauvre en espèces et que senles trois plantes sont risitées de. façon intense : les Cistes, le Sainfoin et la Vigne. D'autre part, certaines colonies ont récolté beaucoup de pollen de P'apaver tandis que d'autres l'ont ignoré totalement. Ce dernier exemple est valable également dans le cas de la récolte du pollen de $Q_{u e r-}$ 
cus ilex. LOUVEAUX (I958) a montré que la récolte du pollen par une colonie représente la résultante de plusieurs facteurs et principalement : la richesse en azote et la présence de substances attractives. D'après ce même auteur, le pollen d'Onobrychis est très attractif et très riche en azote. Or, nous avons récolté au cours de nos essais I 4 p. Ioo de ce pollen bien que cette plante ne soit pas très commune dans la garrigue et qu'elle soit environnée de dizaines d'autres espèces pollinifères très répandues, par exemple le chêne vert et le thym dont chaque pollen représente seulement $2 \mathrm{p}$. Ioo de la récolte. Nous retrouvons là encore une confirmation des travaux de LouvEAUX.

Louveaux (I955) et Chauvin, Louveaux (I956) ont remarqué que les sols incultes étaient, sur l'ensemble du territoire français, les plus productifs pour le pollen. Lors du contrôle d'un grand nombre d'échantillons (en I955) ils ont obtenu des renseignements concernant différentes régions. Nous pourrions essayer de comparer nos résultats aux leurs en ce qui concerne les récoltes de pollen de Cistacées.

Sur la France entière, les Cistes et Hélianthèmes, représentaient 18,03 p. Ioo de la récolte totale (4 $539 \mathrm{~kg}$ ) ; pour le Sud-Est, ils représentaient 37, Io p. Ioo de la récolte; pour la Côte d'Azur, 28,7 p. Ioo ; pour les Pyrénées Orientales, $8 \mathrm{I}, 75$ p. Ioo.

Il est vrai que dans ce département, il s'agissait d'un producteur unique qui avait récolté près de $600 \mathrm{~kg}$ de pollen sur des garrigues très homogènes. Nous avons recueilli 66 p. Ioo de pollen de Cistes au cours de notre essai. D'autre part, depuis I958, nous avons toujours obtenu une grosse récolte sur les garrigues à Cistacées, quelles que soient les conditions météorologiques pendant la floraison. Ceci confirme le caractère des récoltes obtenues par les apiculteurs en I954, I955, I956 sur les Cistes. Nous pensons que ces plantes très communes offrent aux abeilles un pollen abondant dans chaque fleur et facile à collecter. De plus, 1'attractivité de ce pollen pour les abeilles est loin d'être nulle quoique moindre que celle du Sainfoin par exemple. Cet ensemble de facteurs explique en partie le volume important des récoltes qu'il est possible de réaliser sur Cistus albidus.

En ce qui concerne les quantités de pollen que nous avons récoltées, elles sont difficiles à comparer avec celles qui sont citées par les différents auteurs. En effet, les récoltes varient d'une région et d'une année à l'autre et selon le temps pendant lequel les trappes à pollen sont posées. Ce dernier facteur est très important. Les chiffres donnés par HirschFelder, Louveaux et CHAUvin sont très inférieurs à ce que nous avons pu noter.

Chauvin (I955) donne un rendement total par ruche de 3 à $5 \mathrm{~kg}$ sur 5 mois. Cet auteur cite des rendements moyens de $90 \mathrm{~g}$ par jour dans le Jura et un maximum journalier de $450 \mathrm{~g}$ dans les Alpes Maritimes.

HIRSCHFEIDER (I95I) a récolté I $500 \mathrm{~g}$ par quinzaine en juin. LoUvEAUX (T955 et I956) a également relevé des chiffres moins importants que les nôtres dans la région parisienne. Les maxima journaliers obtenus sont de roo g (poids sec). Les récoltes totales varient de moins de $2 \mathrm{~kg}$ à plus de $4 \mathrm{~kg}$ pour toute la saison. L,e maximum journalier indiqué par LouveAux est bien inférieur à ce que nous avons trouvé. Cependant, il entrevoit, dès I954, la possibilité de grosses récoltes si de nombreux facteurs favorables sont réunis. Il explique la récolte de certaines ruches par un phénomène analogue au " hoarding » qui existe chez certains rongeurs. Ces ruches accumuleraient des stocks de pollen très supérieurs à leurs besoins. Quant aux différences quantitatives entre les ruches et qui sont parfois considérables, le développement du 
couvain les explique dans presque tous les cas; cependant, il existe des exceptions pour lesquelles on ne dispose pas d'explication satisfaisante.

Seuls Todd et BIshop (I940) aux U. S. A. trouvent des récoltes qui, quantitativement, pourraient se rapprocher des nôtres.

Rappelons que notre récolte moyenne par ruche et par jour est de I07,5 $\mathrm{g}$ (poids sec) et ceci calculé sur une période de 40 jours avec 37 colonies. Bien entendu, il faut tenir compte du fait que ces chiffres correspondent à la période la plus favorable pour la région et non pas à la récolte de toute la saison. En Ig60, sur 30 colonies, notre moyenne journalière, au même emplacement et dans le même temps avoisinait déjà $80 \mathrm{~g}$ par colonie (poids sec).

\section{5) Divers}

De même que Chauvin (I955) et Louveaux (1958), nous avons retrouvé, sur 5 ans, que les récoltes de pollen pouvaient être abondantes même en dehors de toute miellée et qu'il n'y a aucun rapport entre les deux activités.

Nous avons constaté comme CHAUvin (I955) que les ruches ayant des trappes depuis un certain temps doivent posséder dans leurs rayons un stock de pollen aussi important que les ruches témoins placées dans le même biotope. L'absence de pollen dans les rayons doit être considérée comme anormale et il vaut mieux retirer la trappe.

Enfin, nous ne sommes pas d'accord avec Chauvin et Louvetaux (1956) en ce qui concerne la baisse de rendement des pièges au bout d'un certain temps. En effet, d'après ces auteurs, peu après la pose des trappes supérieures il y a propolisation de la tôle perforée ; les abeilles familiarisées passent alors plus doucement et laissent tomber moins de pelotes dans le tiroir. Nous, n'avons retrouvé nulle part des chiffres permettant une telle interprétation, même pour les ruches ayant des grilles à 6 et à 9 rangs de trous où les abeilles ne sont pas "bousculées " au passage. La récolte crô̂t en fonction de la floraison d: Ciste mais il n'y a jamais baisse de rendement sauf si les lacteurs météorologiques deviennent limitants.

\section{CONCLUSION}

En conclusion, il nous parait possible de donner quelques conseils pratiques pour améliorer la rentabilité de la récolte du pollen :

a) Choisir une région très pollinifère en se souvenant qu'à quelques exceptions près, les terres incultes constituent le milieu naturel le plus favorable ;

b) ne poser des trappes à pollen que sur des ruches fortes possédant au moins $\Upsilon_{5}$ ò̀ $\mathrm{I} 60$ points de couvain (soit une surface de 60 à $64 \mathrm{dm}^{2}$ ) et de bonnes réserves de miel pour le cas où la miellée serait nulle;

c) ne poser les trappes que pendant un temps n'excédant pas 45 jours et à l'époque de la pleine floraison des plantes choisies pour leur pollen ;

d) employer des trappes équipées de tôles perforées ayant trois rangs de trous de $5 \mathrm{~mm}$ de diamètre pour le passage des butineuses. D'autre part, on notera que les grands tiroirs facilitent l'aération du pollen récolté et permettent de réduire le nom- 
bre de déplacements de l'apiculteur. Quel que soit le modèle choisi, ménager si possible une sortie libre à l'opposé de 1'entrée des butineuses dans la trappe pour éviter une " congestion » de la colonie. Si les trappes sont du type "inférieur », éviter l'humidité dans les tiroirs et hausser la ruche par rapport au sol. Enfin, ne pas hésiter à placer tune hausse sur la ruche en cas de miellée, même légère ;

e) si en cours de floraison une colonie manque de pollen dans ses rayons ou présente un tiroir de récolte quasi vide, il faut enlever immédiatement la trappe. Cette ruche est devenue faible, ou orpheline, et présente de toute façon une anomalie.

$$
\text { Reçu pour publication en juillet } 1963 \text {. }
$$

\section{SUMMARY}

\section{EXPERIMENTS WITH A IHIGH PLACED POLLEN TRAP}

Pollen harvesting studied with the aid of traps placed in the upper part of the hives of 40 bee colonies on the "garrigue " of Cistes in the S-E of France gave the following results :

I) the narrower the passage of the trap into the hive, the greater the amount of pollen collected. The optimum, but not the maximum, harvest was obtained with 3 rows of openings into the trap ;

2) an additional free opening into the posterior face of the hive increased the amount of pollen brought in ;

3) the quantity of pollen collected by different bee colonies was practically proportional to the size of their brood. Queenless hives collected pollen, but only in small quantities;

4) among the meteorological factors, wind seemed to exercise the predominating influence ;

5) over a period of 40 days the effects of the removal of pollen on the development of the brood and the weight of the hive were negligible : the former continued to increase in size, while the weight of the latter remained unaffected, except under certain limited conditions ;

6) the bees gathered from the common plants, but they also chose from certain relatively rare plants pollen which was particularly attractive and nutritious;

7) there were indications that pollen gathering was on a much more important scale than had been reported by previous workers.

Finally, practical suggestions were offered for improving the yield of the pollen crop.

\section{RÉFÉRENCES BIBLIOGRAPHIQUES}

Chauvin R., 1955. Réflexions sur la récolte du pollen à l'aide des trappes en i954. L'Apiculteur, I3-21. Chauvin R., Louveaux J., 1956. I.es caractères de la récolte du pollen en 1955. La flore française productrice de pollen pour les abeilles. L'Apiculteur, I 7-28.

Fresnaye J., ig61. Deux nouveaux appareils pour la pesée des ruches. Ann. Abeille, 4, 361-367.

Frestaye J., ig62. Un appareil pour le calcul rapide des surfaces de couvain dans les ruches. Ann. Abeille 5, I 45 -I 53 .

HirSCifelder II., I95I. Quantitative Untersuchungen zum Polleneintragen der Bienenvölker. $Z$. $f$. Bienentorsch., 1, 54, $67-77$.

Louveatx J., I950. Observations sur le déterminisme de la récolte du pollen par les colonies d'abeilles. (Apis mellifica L.). C.R. Acad. Sci., 231, 921-922.

Louveaux J., r954. Études sur la récolte du pollen par les abeilles. L'Apiculteur, 43-50.

Lovveatx J., r955. Introduction à l'étude de la récolte du pollen par les Abeilles (Apis mellifica L.). Physiol. Comp. Écol., 4, I-54.

Louveaux J., 1956. Remarques sur les facteurs conditionnant lo choix par les abeilles (Apis mellifica I..) des plantes leur fournissant du pollen. C.R. Acad., Sci., 242, 2994-2996.

Louveaux J., I958. Recherches sur la récolte du pollen par les abeilles (Apis mellifica L.). Thèse Paris.

Topd F. E., Bismop R. K., r940. Trapping honeybee-gathered pollen and factors affecting yield. $J$. econ. Ent., 33, 866-870. 